Potenziale für eine nachhaltigere Mobilität

\section{Privates Car-Sharing: Die Deutschen teilen ihr liebstes Kind}

\author{
Der Markt der gemeinsamen Autonutzung boomt. \\ Immer mehr Anbieter betreten das Feld - internetgestützte \\ Peer-to-Peer-Plattformen erleichtern das Leihen und Verleihen \\ von Privatfahrzeugen. Was bewegt die Teilnehmenden zum \\ privaten Autoteilen? \\ Von Maike Gossen
}

D as Leitbild „Nutzen statt Besitzen“ zeigt innovative Wege zu einem nachhaltigeren Konsumstil auf. Die Idee dahinter ist einfach: Durch das (Ver-)Leihen, Leasen oder Mieten eines Gegenstandes wird eigentumsloser Konsum ermöglicht. Ökologische Effizienzvorteile - etwa die Erhöhung der Ressourcenproduktivität - können dabei durch eine Nutzungsintensivierung erreicht werden (Scholl 2009: 71f). Auch wenn das zugrunde liegende Prinzip an sich nicht neu ist, er fahren alternative Nutzungspraktiken sowohl in der Forschung als auch in der Praxis wieder verstärkte Aufmerksamkeit. Der Markt der gemeinsamen Autonutzung etwa erlebt derzeit eine unerwartete Dynamisierung. Neue Anbieter sorgen für einen vielfältigen Mobilitätsmix - und erreichen damit Zielgruppen, für die das traditionelle Car-Sharing bisher keine attraktive Alternative zum privaten Autobesitz darstellte. Selbst große Automobilunternehmen setzen mittlerweile auf Car-Sharing. Parallel dazu breiten sich Peer-toPeer-Plattformen für das private Autoteilen aus. Über solche Vermittlungsportale im Internet wird das Verleihen und Leihen privater Fahrzeuge sicher und bequem abgewickelt. Die Betreiber der Plattformen treten dabei als Vermittler zwischen Angebot und Nachfrage auf und bieten die institutionellen Rahmenbedingungen für die gemeinsame Autonutzung, zum Beispiel eine Autozusatzversicherung und einen Überlassungsvertrag.

Die empirische Untersuchung, die im Rahmen der Masterarbeit „Nutzen statt
Besitzen: Motive und Potenziale der internetgestützten gemeinsamen Nutzung am Beispiel des Peer-to-Peer-Car-Sharing“ durchgeführt wurde, ist eine erste explorative Auseinandersetzung mit Nutzer(inne)n des privaten Autoteilens. Konzeptionell orientiert sich die Arbeit an Vorarbeiten zu eigentumslosen Konsumformen (insbesondere Scholl 2009 sowie Scholl und Konrad 2004) und zu Car-Sharing und Mobilitätsverhalten (zum Beispiel Harms 2003, Gaus 2001, Franke 2001). Ausgangspunkt ist die Frage, welche Motive dazu veranlassen, Peer-toPeer-Car-Sharing zu nutzen und welche Rolle dabei ökologische Gründe einnehmen. Diesen Fragen wurde in telefonischen Interviews mit Mitgliedern der Internetplattform „Nachbarschaftsauto“ nachgegangen.

\section{Nutzungsmotive}

Die befragten Leiher(innen) sind in der Regel autolos und erfüllen ihre Mobilitätsbedürfnisse mit einer Kombination aus öffentlichem Nahverkehr und Mietangeboten. Als vorrangigen Grund für die Nutzung des Peer-to-Peer-Car-Sharing führen sie finanzielle Vorteile an. So ist das Leihen eines privaten Fahrzeugs günstiger als zum Beispiel das Mieten über eine konventionelle Autovermietung. Vereinzelt werden auch Umweltschutzgründe angeführt, die jedoch nur in Kombination mit anderen Motiven handlungsrelevant sind. Die Ergebnisse zeigen zudem, dass der Großteil der be- fragten Leiher(innen) eine neue Nutzer (innen)gruppe für das Prinzip Car-Sharing darstellt, da ihre vorherigen Erfahrungen mit Formen der gemeinsamen Autonutzung unerheblich waren.

Auch die Verleiher(innen) partizipieren durch die Teilnahme am privaten Autoteilen zum ersten Mal an Formen des Car-Sharings. Dies ist zunächst nicht überraschend, da sie im Besitz eines eigenen Autos und daher nicht auf andere Car-Sharing-Angebote angewiesen sind. Auch für sie spielen ökologische Gründe eine eher untergeordnete Rolle, andere Motive dominieren. Dazu zählt die Möglichkeit, sich durch das Verleihen des eigenen Autos ein moderates Zusatzeinkommen zu verschaffen und Dritten den Zugang zu individueller Mobilität zu erleichtern. Für diejenigen Verleiher(innen), die über ein ausgeprägtes ökologisches Bewusstsein verfügen, bedeutet die Teilnahme am Peer-to-Peer-CarSharing darüber hinaus eine Art moralische Wiedergutmachung oder Kompensation für den privaten Autobesitz. Sie sind aus beruflichen oder privaten Gründen fest an ein Auto gebunden, wünschen sich langfristig jedoch, einen autolosen Mobilitätsstil zu führen.

Ökologische Motive spielen demzufolge zwar, ähnlich wie es auch für das traditionelle Car-Sharing konstatiert werden kann, für beide Seiten eine Rolle, sie dominieren jedoch die Motivlagen nicht und werden höchstens in Kombination mit anderen Gründen als handlungsleitend beschrieben.

\section{Langfristige Potenziale des Peer-to-Peer-Car-Sharings}

Für die befragten Leiher(innen) ist Peer-to-Peer-Car-Sharing als temporäre Lösung interessant, da es zur jeweiligen Lebenssituation und den damit einhergehenden Mobilitätsanforderungen passt. Sollten sich die persönlichen Lebensumstände in Zukunft verändern und sich dadurch auch die Mobilitätsanforderungen wandeln, etwa durch einen Umzug in eine weniger gut durch öffentliche Verkehrsmittel erschlossene Gegend, ist für einige Leiher(innen) die (Wieder-)An- 
schaffung eines eigenen Autos vorstellbar. Für sie ist die Verkehrsmittelwahl vor allem das Resultat pragmatischer Abwägungen und nicht Ausdruck ihrer Umweltschutzorientierung oder ideeller Überzeugungen.

Dass die Teilnahme am Peer-to-PeerCar-Sharing eher eine vorübergehende Wahl ist, kann gleichermaßen für die Gruppe der Verleiher(innen) angenommen werden. Auch wenn sich ihr Verhältnis zum Auto größtenteils als pragmatisch und zweckorientiert beschreiben lässt, würde bei manchen die Anschaffung eines neuen und - verglichen mit dem derzeitigen - wertvolleren Autos mit großer Wahrscheinlichkeit zur Aufgabe des Peer-to-Peer-Car-Sharing führen.

\section{Ausblick}

Die Untersuchung konnte deutlich machen, dass die Angebote des Peer-toPeer-Car-Sharings neue Nutzer(innen)gruppen für die gemeinsame Autonutzung erschließen können. Aufseiten der Leiher(innen) kann dadurch das Eigentum eines eigenen Autos substituiert werden, was positive ökologische Umwelteffekte zur Folge hat. Für Verleiher(innen) bietet sich eine Gelegenheit, ihr im eigenen Alltag wenig genutztes Auto stärker auszulasten und gleichzeitig anderen Personen bedarfsweise Zugang $\mathrm{zu}$ individueller Automobilität zu ermöglichen.

Gleichwohl bleibt offen, ob Peer-toPeer-Car-Sharing als Alternative zum eigenen Auto langfristig Bestand hat. Vor diesem Hintergrund sind innovative Weiterentwicklungen des Konzepts interessant, die aufzeigen, wie sich das private Autoteilen weiter vereinfachen und noch zielgruppengerechter gestalten lässt. In Belgien teilt sich beispielweise eine Gruppe von Menschen ein Auto, das sich nicht mit einem Schlüssel sondern per Smartphone-App öffnen lässt. Dadurch entfällt die aufwändige Planung und Durchführung der persönlichen Schlüsselübergabe.

In welchem Maße die gemeinsame Autonutzung und insbesondere das Peerto-Peer-Car-Sharing zu einem nachhaltigeren Mobilitätsverhalten beitragen kön- nen, bleibt eine interessante Frage. Nicht zuletzt die vor allem bei Jüngeren zu beobachtende Abkehr vom Privatauto als Statussymbol (Terporten et al. 2012) wird die Zukunft des Car-Sharing wesentlich beeinflussen. Sowohl in den neueren Angeboten, die durch den Einsatz kommunikationstechnischer Innovationen und Praktikabilität punkten, als auch in der zunehmend verbreiteten Haltung unter Konsument(inn)en, dass umwelt- und klimaschonendes Handeln zeitgemäß sei (Hunsicker et al. 2008: 23), besteht eine Chance für die weitere Diffusion umweltfreundlicher Mobilitätsweisen wie des Car-Sharings.

\section{Literatur}

Franke, S. (2001): Car-Sharing: Vom Ökoprojekt zur Dienstleistung. Berlin, Edition Sigma.

Gaus, H. (2001): Entwicklung eines Bezugsrahmens zur Untersuchung des ökologieorientierten Mobilitätsverhaltens. Chemnitz, Fakultät für Wirtschaftswissenschaften an der Technischen Universität Chemnitz.

Harms, S. (2003): Besitzen oder Teilen: Sozialwissenschaftliche Analyse des Car Sharings. Zürich, Rüegger.

Hunsicker, F. et al. (2008): Megatrends und Verkehrsmarkt: Langfristige Auswirkungen auf den Personenverkehr. InnoZ-Bausteine. Berlin, Innovationszentrum für Mobilität und gesellschaftlichen Wandel (InnoZ) GmbH.

Scholl, G. (2009): Marketing nachhaltiger Dienstleistungen. Bedingungen der Übernahme und Empfehlungen zur Vermarktung von eigentumsersetzenden Konsumpraktiken. Marburg, Metropolis.

Scholl, G. / Konrad, W. (2004): Verbraucherakzeptanz von Nutzungsstrategien. Diskussionspapier des IÖW. Berlin/Heidelberg, Institut für ökologische Wirtschaftsforschung (IÖW).

Terporten, M. et al. (2012): Veränderte Kundenwünsche als Chance zur Differenzierung. In: Proff, H. et al. (Hrsg.): Zukünftige Ent wicklungen in der Mobilität - Technische und betriebswirtschaftliche Aspekte. Wiesbaden, Gabler Verlag.

\section{AUTORIN+ KONTAKT}

Maike Gossen ist wissenschaftliche Mitarbeiterin im Forschungsfeld Ökologischer Konsum am Institut für ökologische Wirtschaftsforschung. Die dem Beitrag zugrunde liegende Masterarbeit wurde jüngst als IÖW Schriftenreihe 202/12 veröffentlicht.

Institut für ökologische Wirtschaftsforschung (IÖW), Potsdamer Straße 105, 10785 Berlin, Tel.: +49 30 884594-0, E-Mail: maike.gossen@ioew.de

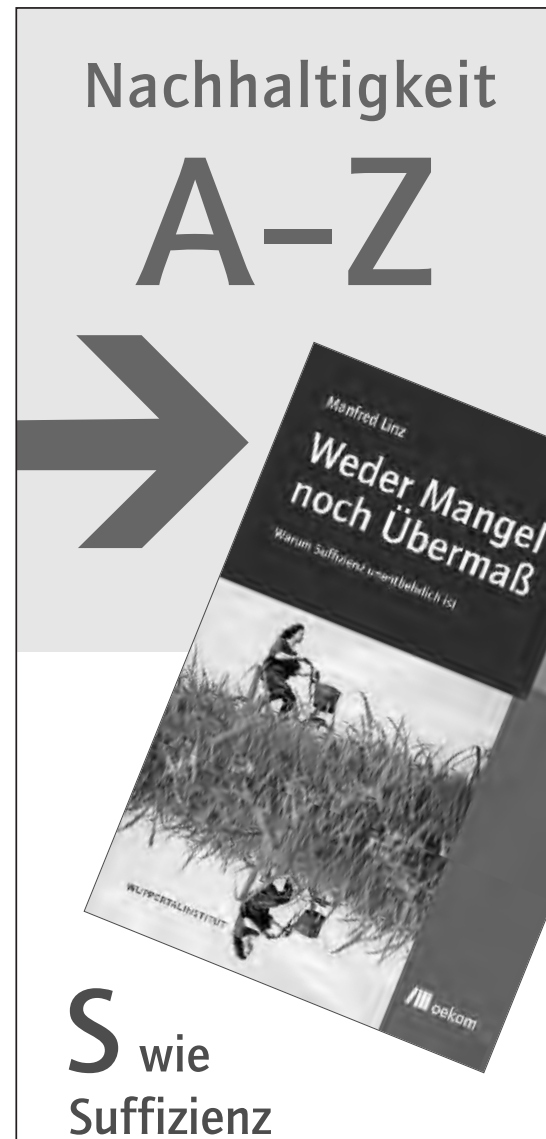

Effizienzsteigerungen allein reichen nicht aus, um Ressourcenverbrauch und $\mathrm{CO}_{2}$-Ausstoß zu senken. Wir müssen unser Konsumverhalten insgesamt überdenken.

In seinem Buch "Weder Mangel noch Übermaß. Warum Suffizienz unentbehrlich ist" erklärt Manfred Linz nicht nur, warum wir einen genügsameren Lebensstil pflegen sollten. Er zeigt auch, dass mehr Suffizienz auch mehr Gerechtigkeit bringt - und beschreibt anschaulich ihre praktische Umsetzung.

\section{Linz}

Weder Mangel noch Übermaß Warum Suffizienz unentbehrlich ist

146 Seiten, broschiert, 19,95 Euro, ISBN 978-3-86581-399-2

Erhältlich bei

www.oekom.de

oekom@verlegerdienst.de

\section{/II oekom}

Die guten Seiten der Zukunft 
Copyright (C) 2013, IÖW und oekom Verlag. Die Nutzung des Artikels ist Abonnenten von Ökologisches Wirtschaften vorbehalten. Nachdruck und Vervielfältigung des Artikels einschließlich Speicherung und Nutzung auf optischen und elektronischen Datenträgern nur mit Zustimmung der Redaktion von Ökologisches Wirtschaften (http://www.oekologischeswirtschaften.de). 